Ann. Génét. Sél. anim., I969, 1 (4), 383-390.

REVUE BIBLIOGRAPHIQUE

\title{
LA DÉTERMINATION DU SEXE CHROMOSOMIQUE DANS L'ÉTUDE DE L'INTERSEXUALITÉ ASSOCIÉE A L'ABSENCE DE CORNES CHEZ LA CHÈVRE D'ORIGINE ALPINE
}

\author{
Françoise HULOT et Parvathi-K. BASRUR (*) \\ Station centrale de Génétique animale, \\ Centre national de Recherches zootechniques, 78-Jouy-en-Josas \\ Institut national de la Recherche agronomique \\ * Department of biomedical Sciences \\ University of Guelf, Ontario - Canada
}

\section{RÉSUMÉ}

Dans les troupeaux de chèvres mottes, les résultats les plus récents acquis par examen direct de caryotypes sur culture cellulaire vérifient les conclusions auxquelles aboutissaient les études génétiques : les intersexués et les mâles stériles par hypoplasie des testicules (ou pseudomâles) sont des femelles génétiques, les mâles stériles par blocage de l'épididyme, avec parfois quelques caractéristiques intersexuées, sont de vrais mâles.

\section{I. - INTRODUCTION}

Dans les races caprines originaires des Alpes, les anomalies telles que l'intersexualité et la stérilité des mâles sont fréquentes et associées au caractère "absence de cornes ". L'étude de la transmission héréditaire des différents défauts du tractus génital a conduit les généticiens à prédire le sexe des anormaux (LAUVERGNE, 1969). Afin de vérifier l'exactitude des pronostics, la connaissance de la formule sexuelle s'avérait indispensable. Deux types de travaux ont alors été entrepris : recherche sous forme virtuelle du chromosome $X$ dans les noyaux quiescents (tests cytologiques), identification des hétérosomes dans la cellule en division (test caryologique). Ils sont décrits dans une première partie destinée à rassembler toutes les données cytologiques acquises au cours des temps, puis discutés dans une seconde partie qui tend à une synthèse des connaissances du sexe génétique des anormaux. 
F. HUL,OT, P.-K. BASRUR

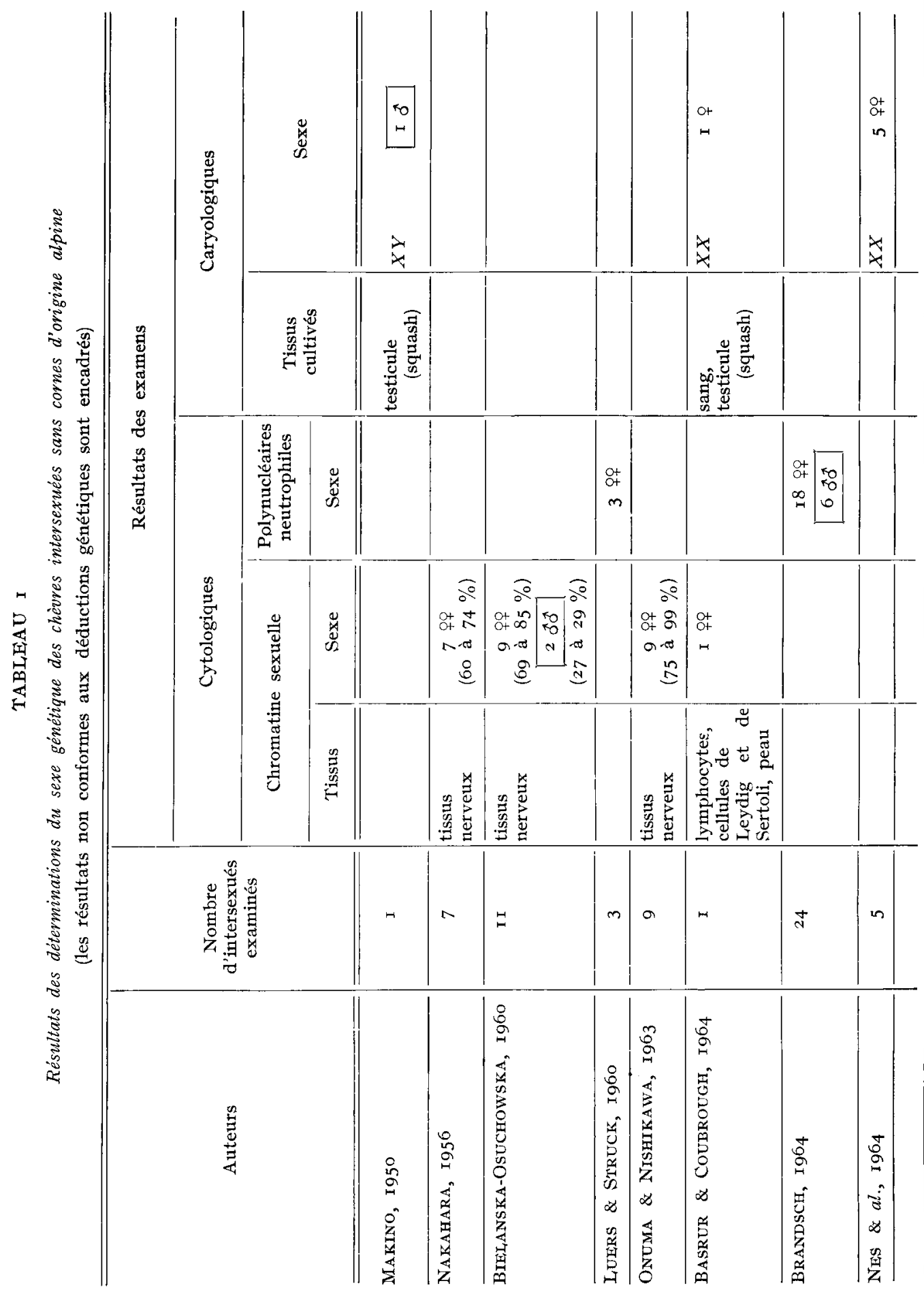


SEXE CHROMOSOMIQUE DES CHÈVRES INTERSEXUÉES

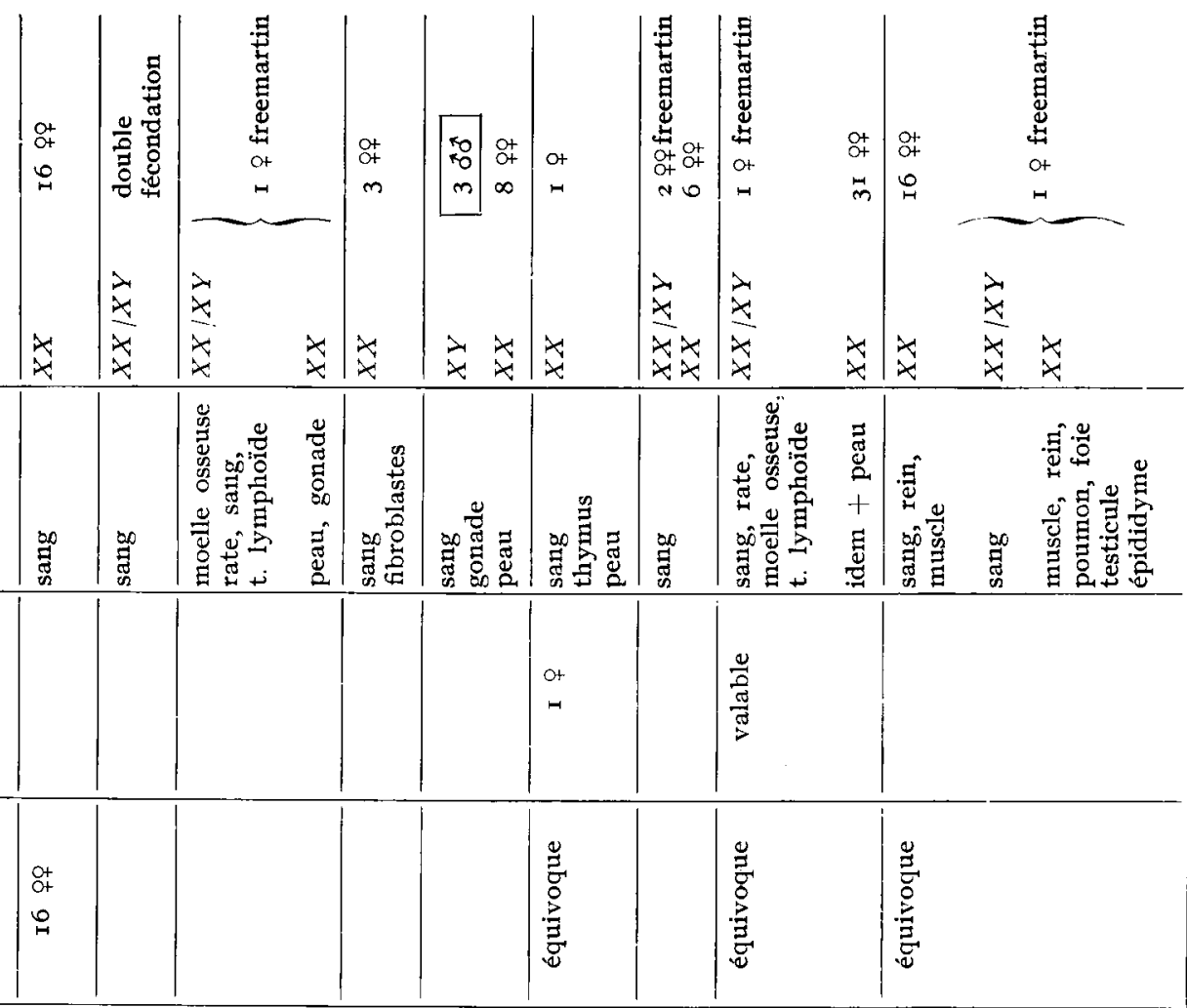

\begin{tabular}{|c|c|c|c|c|c|c|c|c|}
\hline 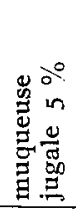 & & & & & 葛泀 & & 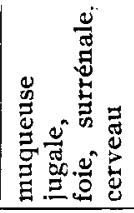 & 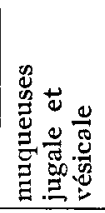 \\
\hline$\stackrel{\circ}{\circ}$ & $r$ & $H$ & $m$. & $\Xi$ & 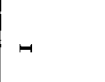 & $\underset{\infty}{\underline{E}}$ & W & $\hat{F}$ \\
\hline 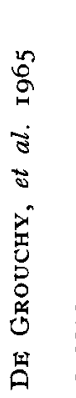 & 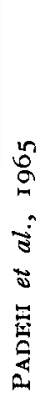 & 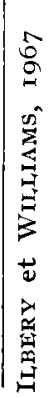 & 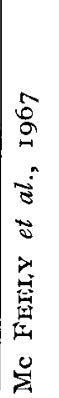 & 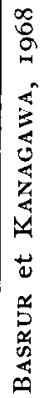 & 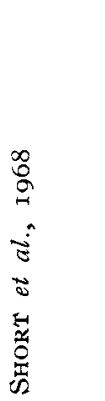 & 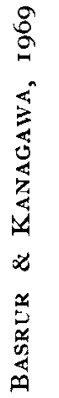 & 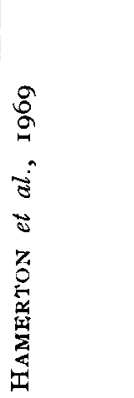 & 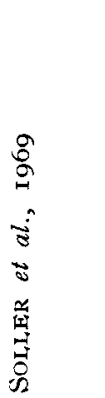 \\
\hline
\end{tabular}




\section{II. - RÉSULTATS}

\section{A. - Recherches cytologiques}

Les tests cytologiques dont les résultats étaient, entre 1956 et 1964, utilisés comme critères de définition du sexe de la chèvre, ne représentent plus maintenant qu'un complément. aux techniques caryologiques. L'ensemble des travaux est rapporté dans les tableaux 1 et 2 .

\section{La chromatine sexuelle (ou corpuscule de BARR).}

Cette masse de chromatine, représentant un des chromosomes $X$ dans les noyaux femelles, est apparente en particulier dans les tissus nerveux avec une fréquence de 70 à $90 \%$ (BARR et Bertram, 1949). Nakahara (1956) et Onuma et Nishikawa (1963) ont établi sur 7 et 9 intersexués respectivement des fréquences voisines de celles caractérisant les femelles normales. Par contre, Bielanska-Osuchowska (1960) détectait 2 mâles génétiques sur 11 intersexués examinés.

D'autres types cellulaires ont, par la suite, été utilisés, mais les résultats obtenus sont moins nets. L'observation d'épithélium jugal par De Grovchy et al. (1964) et l'examen des lymphocytes, de cellules de Leydig, de Sertoli et épithéliales par Basrur et Coubrovgh (1964) ont permis de confirmer le sexe femelle de nombreux intersexués. Par contre, les tests de Short et al. (1968), Hamerton et al. (1969) sur cellules hépatiques, surrénaliennes, nerveuses et de Soller et al. (1969) sur des frottis d'épithéliums buccal et vésical sont trop peu clairs pour permettre une interprétation valable.

\section{La détermination sexuelle hématologique.}

Davidson et Smith (1954) ont défini des caractéristiques morphologiques des noyaux des polynucléaires neutrophiles. Trois groupes principaux d'excroissances nucléaires sont identifiés : des appendices pédiculés - $A-$ (drumsticks), caractéristiques de la femelle, des appendices sessiles $-B-$ (sessile nodules) plus fréquents chez les femelles que chez les mâles, des petits clubs $-C-$ (small clubs) dominants chez le mâle. Certains auteurs déterminent le sexe d'un individu en évaluant le rapport $\frac{A+B}{C}$. Brandsch et Erdman (1960) se sont intéressés plus particulièrement aux appendices $A$ et, testant la méthode sur 30 caprins normaux des deux sexes, ont considéré comme satisfaisante l'identification de 4 de ces éléments chez un individu pour le juger "génétiquement femelle ". Luers et Struck (1960) par ces procédés ont attribué le sexe femelle à 3 intersexués et Brandsch (1964) a réparti 24 intersexués en 18 femelles et 6 mâles. Analysant également 5 mâles stériles, ce dernier auteur note les résultats suivants : 2 femelles (hypoplasie testiculaire), 3 mâles (1: hypoplasie testiculaire, 2 : épididyme anormal). Pour Hamerton et al. (1969), Short et al. (1968) et Soller et al. (1969), les résultats obtenus par cette méthode corroboraient leurs recherches caryologiques.

\section{B. - Recherches caryologiques}

Le caryotype de la chèvre comprend 29 paires d'autosomes acrocentriques. Le chromosome $Y$, métacentrique très petit, est parfaitement décelable depuis les travaux de BASRUR et STOLZ (1967). Le chromosome $X$ doit être identifiable à l'un des plus longs acrocentriques (1 ${ }^{\mathrm{er}}, 2^{\mathrm{e}}$ ou $3^{\mathrm{e}}$ ?) et posséderait, selon Evans (1964), un bras très court qu'il est encore difficile de mettre en évidence dans toutes les préparations.

La plupart des travaux de caryologie sur l'intersexualité caprine sont récents (à partir de 1964) et utilisent la technique moderne de culture cellulaire, en particulier celle des leucocytes sanguins. L'examen des chromosomes sexuels ainsi obtenus a permis un diagnostic très rigoureux du sexe des intersexués ( $X X$ et $X Y$ dans quelques cas) et des "mâles stériles " ( $X X$ et $X Y$ ). Par ailleurs, il a rendu possible la détection de chimères $X X / X Y$ que les tests cytologiques n'avaient pas mis en relief. Le détail de ces résultats est colligé dans les tableaux 1 et 2 . 


\section{TABLEAU 2}

Résultats des déterminations du sexe génétique des máles stériles sans cornes observés dans les races de chèvres d'origine alpine

(les résultats non conformes aux déductions génétiques sont encadrés)

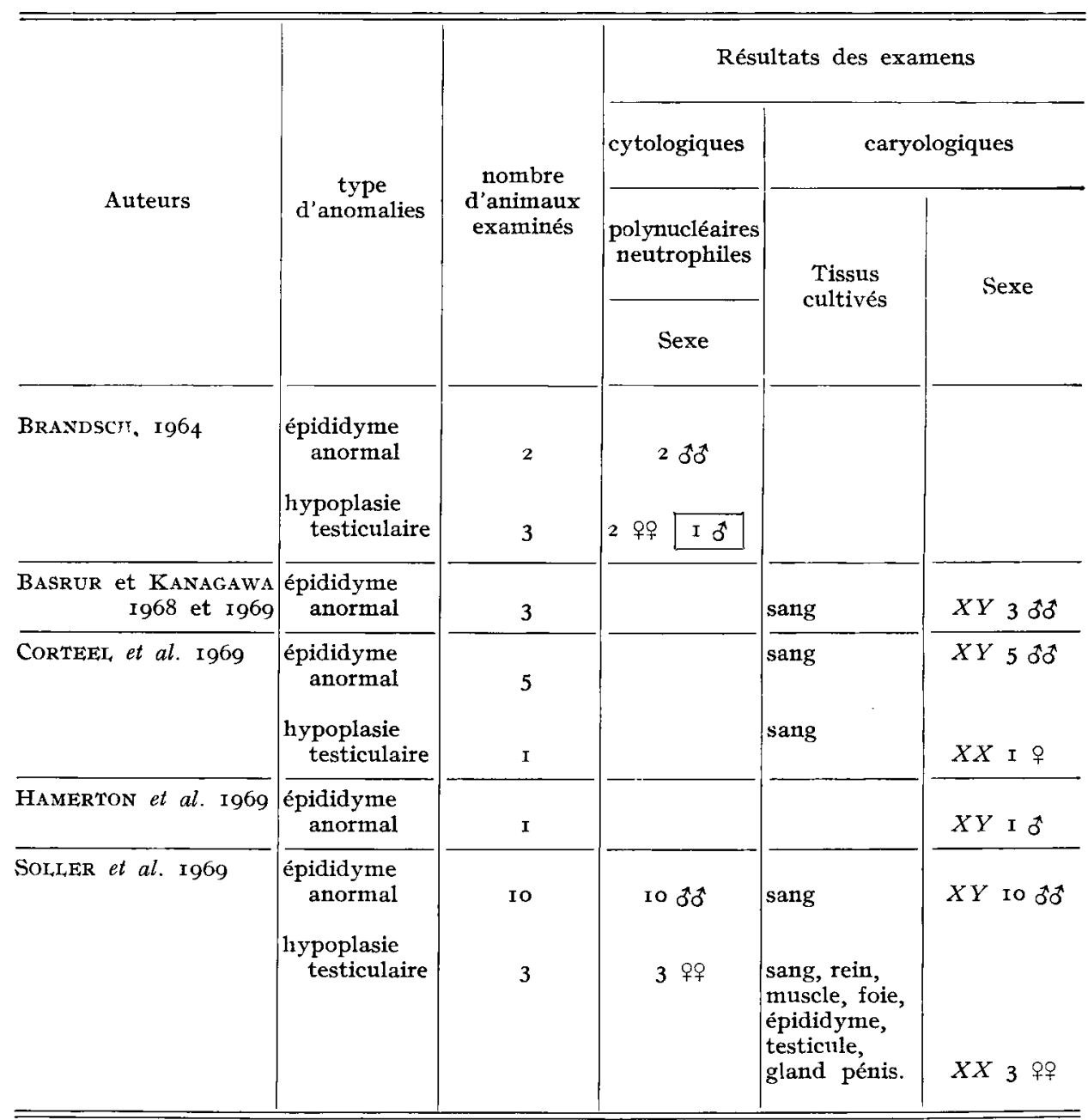

\section{III. - DISCUSSION}

\section{A. - Le sexe génétique des intersexués}

- L'examen des données de ségrégation qui accusaient un fort déficit en femelles dans les populations sans cornes avait conduit les généticiens à faire l'hypothèse que les intersexués étaient des femelles génétiques homozygotes pour le gène motte. Les résultats de la détermination du sexe par les tests cytologiques et caryologiques en vérifient le bien-fondé. En effet, sur un effectif global de 151 individus, 133 sont effectivement des femelles génétiques. 
- Cependant, la présence inexplicable de quelques individus "mâles " doit être discutée. L'examen, déjà ancien, de MAKino (1950), s'appuie sur l'observation de préparations sommaires qui rendent contestable l'identification des hétérosomes. Les résultats divergents de Brandsch, établis sur la présence des drumsticks, ne sont pas d'une rigueur absolue. L'auteur lui-même pense que cette technique n'est pas sûre et ne croit pas "impossible que tous les intersexués appartiennent au sexe femelle selon leur statut chromosomique ".

BASRUR et Kanagawa, dans leur communication préliminaire de 1968, rapportent l'existence de 3 chèvres "intersexuées " $X Y$ sur 11 examinées. Cette assertion, fondée sur des données caryologiques, concourt à la confusion générale des interprétations cytogénétiques de l'intersexualité. Les descriptions anatomiques données en 1969 sur ces mêmes animaux, soulèvent le problème de leur exacte terminologie. En effet, par certains aspects, ces 3 "intersexués" méritent d'être inclus dans la catégorie des "mâles stériles " avec défauts épididymaires décrits par Soller et al. (1969). Cependant, ces mêmes animaux exhibent quelques signes certains d'intersexualité tels que l'absence de pénis et la présence d'un utérus.

- Par ailleurs, les individus $X X / X Y$ sont également des femelles génétiques mais leur intersexualité est indépendante du caractère motte et attribuable au freemartinisme, phénomène connu dans d'autres espèces. Seul l'animal de PADEH et al. (1965), né unique, proviendrait de la fécondation dispermicque d'un ovule digynique. Selon différents auteurs, la proportion de ces individus chimères ne représente qu'un faible pourcentage parmi les intersexués. Le gène motte $P$ reste la principale source de trouble du développement sexuel des femelles homozygotes.

\section{B. - Le sexe génétique des mâles stériles}

Des travaux déjà anciens d'auteurs allemands avaient conduit à la distinction anatomique de deux types de mâles stériles, les uns caractérisés par une hypoplasie des testicules, les autres apparemment normaux mais atteints d'un blocage du transit épididymaire. L'étude de la transmission de cette anomalie, également associée au gène motte $P$, a abouti à l'élaboration de 2 hypothèses : les premiers seraient des femelles génétiques $P P$ à masculinisation poussée, les seconds des mâles génétiques $P P$.

Les résultats de Brandsch (1964) ne vérifient que partiellement cette hypothèse mais la relative validité de la technique utilisée a déjà été exposée. Toutes les études caryologiques portant sur un total de 4 mâles à hypoplasie testiculaire et 19 mâles à atteintes épididymaires. corroborent parfaitement les pronostics génétiques.

Les problèmes de terminologie des "mâles stériles" rejoignent ceux évoqués à propos des intersexués. Ainsi les individus $X Y$ de Basrur et Kanagawa, discutés précédemment, semblent devoir être classés dans ce chapitre. Par ailleurs, certains "intersexués " $X X$ de Hamerton et al. (1969) seraient susceptibles d'être qualifiés par d'autres auteurs de "mâles stériles ".

\section{IV. - CONCLUSION}

Les examens cytologiques et caryologiques confirment dans l'ensemble les hypothèses génétiques. Des imprécisions subsistent encore en ce qui concerne l'anatomie des diverses catégories d'anormaux, les définitions entre l'intersexualité et les différentes formes de stérilité mâle devant être totalement révisées. Il serait souhaitable, sur les produits de croisements mottes contrôlés génétiquement, de réaliser simultanément des études d'anatomie et histologio génitales rigoureuses, de biochimie hormonale sexuelle et de caryologie. 


\title{
SUMMARY
}

\author{
GENETICAL SEX DETERMTNATION IN THE STUDY OF INTERSEXUALITY \\ IINKED WITH HORNLESSNESS IN THE GOAT OF ALPINE ORIGIN
}

The object of this report is to gather together and to discuss the cytogenetic knowledge amassed so far on sex anomalies associated with the polled trait in goats.

Studies on intersexuality and male sterility have led anatomists and geneticists to make several predictions on the genetic sex of the abnormal animals. In order to verify these predictions, the exact nature of the sex anomaly in the affected goats and their cytogenetic sex have to be ascertained. Earlier, cytogenetic tests relied entirely on such parameters as the sex chromatin of the interphase nuclei and the drumstick of the polymorphonuclear neutrophils. Since 1964, a more reliable approach to the diagnosis of genetic sex has been followed through the use of cell culture and more frequently blood culture method for the deleniation of sex chromosomes in metaphase spreads.

The results of all these studies, summarized in the tables, support the predictions of geneticists on goats with sex anomalies. A majority of the intersexes (133 out of 151 cases examined) were genetic females on the basis of their cytological sex determining mechanism. Some intersex goats were "freemartins "as confirmed by chimerism for $X X$ and $X Y$ cells in the hemopoietic tissues and a few were genetic males with $X Y$ type sex complements. "Male " goats with sex anomalies include at least 2 distinct categories : the $X \boldsymbol{X}$ individuals with testicular hypoplasia (pseudo-males) and $X Y$ individuals with epididymal defects and /or varying degrees of intersexual modifications such as the absence of a penis and the presence of a uterus.

Some degree of confusion still exists regarding the identification and grouping of the abnormal animals in these categories on the basis of anatomic criteria. The ambiguity in the literature regarding the cytogenetic and anatomic sex of the affected goats may be attributable to the lack of common anatomic criteria for distinguishing "intersexes " from "pseudo-males " and "sterile males".

\section{RÉFÉRENCES BIBLIOGRAPHIQUES}

BARR M.L., Bertram E.G., I949. A morphological distinction between neurones of the male and female, and the behaviour of the nucleolar satellite during accelerated nucleo-protein synthesis. Nature, I,ond,, 163, $676-677$.

Basrur P.K., Coubrough R.I., I964. Anatomical and cytological sex of a Saanen goat. Cytogenetics, 3, $4 \mathrm{I} 4-426$.

Basrur P.K., Kanagawa H., 1968. Cytogenetic studies on intersex goats. (abstr.) Canad. J. Genet. Cytol., 10, 764 .

BASRUR P.K. et Kanagawa H., I969. Anatomic and cytogenetic studies on r9 hornless goats with sexual disorders. Ann. Génét. Sél. anim., 1, 349-378.

Basrur P.K., Stoltz D.R., 1967. The $\mathrm{Y}$ chromosome of the Goat. J. Hered., 58, $26 \mathrm{I}-262$.

Brelanska-OSuchowska Z., 1960. Hermaphroditism in goat. III. Cytogenetic sex diagnosis in hermaphrodite goats (en polonais). Medycyna wet., 6, 658-662.

BRANDSCH H., r964. Vergleichende Untersuchungen zur Vererbung des Kryptorchismus und der Intersexualtät bei Haustieren. Kühn-Arch., 77, 323-425.

BRANDSCH H., ERDMAN S., I960. Kernmorphologische Untersuchungen zur Geschlechtserkennung bei Ziegen. Arch. Geflugelz. Kleintierk., 9, г74-181.

Corteel J.M., Hulot Françoise, Courot M., Atral J., Philippon A., I969. Fxamens morphologiques, caryologiques, physiologiques et pathologiques de boucs stériles sans cornes. Ann. Génét. Sél. anim., 1, $34 \mathrm{I}-348$.

Davinson E.M., SMITH D.R., 1954. Sex differences in polymorphoneutrophil leucocytes. Br. med. $J$., 2, 6-7.

EvaNs H.J., r965. A simple microtechnique for obtaining human chromosome preparations with some comments on DNA replication in sex chromosomes of the Goat, Cow and Pig. Expl. Cell. Res., 38, 5 II-5I6.

Grouchy J. de, IAAuvergne J.-J., Ricordeato G., I965. Études cytogénétiques chez i6 chèvtes intersexuées. C.R. hebd. Séanc. Acad. Sci., Paris, 260, 2932-2935.

Hamerton J.I.., Dickson M., Pollard C.E., Grieves S.A., Short R.V., I969. Genetic intersexuality in goats. J. Reprod. Fert., Supplem., 7, 25-5I.

Hulot Françoise, L,AUVERGNe J.-J., I967. L,es chromosomes des Ruminants. Ann. Génét., 10, 86-97. ILBERY P.I.T., WILLIAMS D., I967. Evidence of the freemartin condition in the Goat. Cytogenetics. 6, $276-285$. 
LaUVERGNe J.-J., I969. Progrès des connaissances génétiques sur l'intersexualité associée à l'absence de cornes chez la Chèvre domestique. Ann. Génét. Sél. anim., 1.

LuERS T., STRuck E., I96o. Untersuchungen zur Geschlechtspezifischen Struktur der Neutrophilenkerne bei einigen Haustieren (Ziege, Schaf, Schwein), unter Berïcksichtigung der Zwitter. Zool. Anz., 164, 89-103.

Mc Feely R.A., Hare W.C.D., Biggers J.D., I967. Chromosomes studies in I4 cases of intersex in domestic animals. Cytogenetics, 6, 242-253.

Maxino S., I950. Constitution of the sex chromosome in an intersex goat. Pap. coord. Com. Res. Genet., $1, \mathbf{1}-3$.

Nakahara T., 1956. Studies on the sex chromatin in cell nuclei of the Goat. I. Detection of the sex chromatin in nerve cells. (en japonais). Jap. J. anim. Reprod., 2, 69-72.

Nes N., Andersen K., Stagsvorp P., I963. Chromosomes de chèvres hermaphrodites (en norvégien). Medlemsbl. Norske Veterinaerfor., 7, I 55 -166.

Onuma H., Nishrkawa Y., I963. The sex chromatin of domestic animals. III. On the genetic sex of bovine freemartin and goat intersex. Bull. nation. Inst. anim. Industr. Jap., 2, 277-286.

Padeh B., Wysoki M., Ayalón N., Soller M., I965. An XX/XY hermaphrodite in the goat. Israel J. med. Sci., 1, roo8-ror 2.

Short R.V., Hamerton J.L., Grieves S.A., Pollard C.E., I968. An intersex goat bilaterally asymmetrical reproductive tract. J. Reprod. Fert., 16, 283-29r.

Soller M., Padeh B., Wysoki M., Ayalon N., I969. Cytogenetics of Saanen goats showing abnormal development of the reproductive tract associated with the dominant gene for polledness. Cytogenetics, $8,5 \mathrm{r}-67$. 